\title{
Differential susceptibility to noise of mixed Turing and Hopf modes in a photosensitive chemical medium
}

\author{
S. Alonso ${ }^{1}$, D. G. Míguez ${ }^{2}$ and F. Sagués ${ }^{3}$ \\ ${ }^{1}$ Abteilung Physikalische Chemie, Fritz-Haber-Institut der Max-Plank-Gesellschaft-14195 Berlin, Germany \\ ${ }^{2}$ Department of Systems Biology, Harvard Medical School - 02115 Boston, MA, USA \\ ${ }^{3}$ Departament de Química Física, Universitat de Barcelona - 08028 Barcelona, Spain
}

received 5 October 2007; accepted in final form 27 November 2007

published online 31 December 2007

PACS 05.40.Ca - Noise

PACS 82.40.Ck - Pattern formation in reactions with diffusion, flow and heat transfer

PACS 47.54.-r - Pattern selection; pattern formation

\begin{abstract}
We report on experiments with the photosensitive chlorine dioxide-iodine-malonic acid reaction (CDIMA) when forced with a random (spatiotemporally) distributed illumination. Acting on a mixed mode consisting of oscillating spots, close enough to the Hopf and Turing codimension-two bifurcation, we observe attenuation of oscillations while the spatial pattern is preserved. Numerical simulations confirm and extend these results. All together these observations point out to a larger vulnerability of the Hopf with respect to the Turing mode when facing noise of intermediate intensity and small correlation parameters.
\end{abstract}

Copyright (C) EPLA, 2008

During these last decades it has been fully recognized that fluctuations, either internal (thermal) or parametric can conspire with nonlinearities to enhance regular behavior. Examples range from electronic and optical devices to chemical reactions or biophysical systems, as recently summarized in [1]. In time evolving systems, noise-anticipated periodicities are known to result from a phenomenon known as coherence resonance (or stochastic coherence as we prefer to call it) [2]. In extended systems, noisy precursors of steady patterns may appear below but close enough to a spatially symmetry-breaking instability [3]. A still never addressed question refers to the role of noise in the neighborhood of higher-order bifurcations, for instance a Turing/Hopf coexistence. Below the corresponding codimension-two point, the relevant question is whether a particular mode, either a global oscillation, a patterned static state or some mixed solution, would be selectively anticipated under noise forcing. Above the bifurcation, the principal interest lies, conversely, in the role of fluctuations in making one of these particular modes dominant over the rest. Adopting this latter view we report on observations of a mixed oscillating patterned state losing stability to a pure spatial mode under distributed noise of intermediate intensity and small values of the correlation parameters.

Chemical systems are natural candidates to investigate effects of noise superimposed on modes of self-organization [4]. This is particularly true for photosensitive reactions, where the illumination intensity acts as an easily tunable control parameter. The prototypical example is the Belousov-Zhabotinsky (BZ) reaction, where spatially distributed noise has been demonstrated to drive [5], sustain [6] or organize waves [7]. The somewhat alternative, still of photosensitive nature, is the chlorine dioxide-iodine-malonic acid mixture (CDIMA), well known to give rise to spatial Turing patterns [8].

The CDIMA reaction, other than Turing patterns, is capable as well to sustain global oscillations [9] and forced spatio-temporal resonances [10-12]. In addition, we recently reported observations of mixed Turing/Hopf modes close to codimension-two conditions [13]. In this situation, the CDIMA sample displays a hexagonal static lattice of spots synchronously oscillating in amplitude. Here, we pursue experimentally and numerically the study of this regime by addressing the situation of a Turing-Hopf coexistence when subjected to the disturbing effect of a spatiotemporal noise. The influence of distributed fluctuations is interpreted in terms of a differential noise susceptibility of each mode under such degenerated conditions. Our work extends previous studies of noise-anticipated patterns close to the pure Turing instability of the CDIMA reaction under disorderly frozen illuminating conditions (static noise) [14]. Numeric results based on the LengyelEpstein (LE) model [15] have been also published recently 


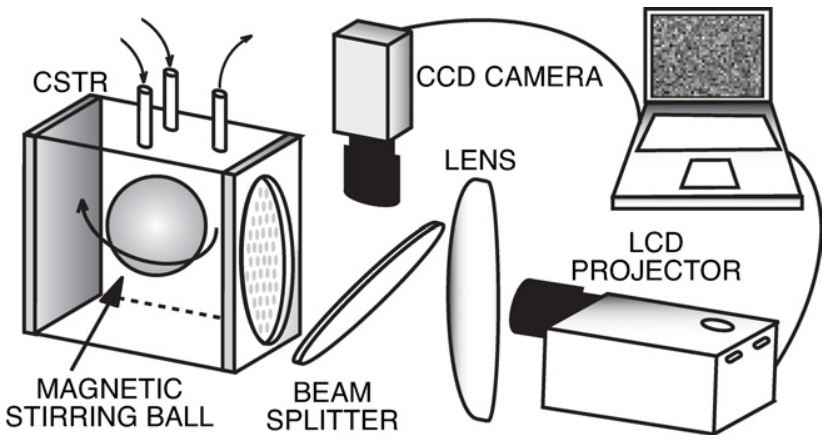

Fig. 1: Scheme of the experimental setup for noise forcing of the CDIMA reaction.

for spatio-temporal Gaussian fluctuations [16,17]. In a rather different context and, actually, as a result of a global random alternation between a pair of pattern-free dynamics, Turing-like structures have been predicted to occur for morphogenic models [18] and in a FitzHugh-Nagumo dynamics [19].

Experiments were performed with the photosensitive CDIMA reaction with input chemical concentrations $\left[\mathrm{I}_{2}\right]_{0}=0.45 \mathrm{mM}, \quad\left[\mathrm{ClO}_{2}\right]_{0}=0.1 \mathrm{mM}, \quad[\text { malonic acid }]_{0}=$ $0.9 \mathrm{mM}$, $[\text { poly }(\text { vinyl alcohol })]_{0}=0.5 \mathrm{~g} / \mathrm{L}$ and $\left[\mathrm{H}_{2} \mathrm{SO}_{4}\right]_{0}=$ $10 \mathrm{mM}$. Figure 1 is a schematic representation of the setup used, which is described in more detail in [12]. Reaction occurred in a continuously stirred tank reactor (CSTR). Patterns are formed in a $2 \%$ agarose gel inside the CSTR, and observerd and recorded through an optical window with a CCD (JAI M50 high resolution) camera connected to a computer. Applied spatio-temporal noise consisted of a computer-generated pattern of square pixels with random Gaussian distributed intensities in an eight-bit gray scale between 0 and 255 (mean value 128 and variance 35 ) and further projected onto the gel (LCD Hitachi, CP-X327 projector). White-light illumination intensity ranged from $1100 \pm 50 \times 10^{-6} \mathrm{~W} / \mathrm{cm}^{2}$ (corresponding to 0 in gray level) to $19800 \pm 50 \times 10^{-6} \mathrm{~W} / \mathrm{cm}^{2}$ (corresponding to 255 in gray level). The chemical reactor was inmersed into a thermostated water bath at $4^{\circ} \mathrm{C}$ continuously stirred to avoid possible local increases in temperature due to illumination. Synchronization between projection and image acquisition processes allowed to turn off the projection for a very short period of time (negligible compared with the time scales of the chemical system) to take a snapshot of the pattern without disturbing it. To be close but above the Turing/Hopf codimension-two point we chose an averaged illumination $I=(10500 \pm 50) \times 10^{-6} \mathrm{~W} / \mathrm{cm}^{2}$. Under this reference level, i.e. in the absence of noise, the system exhibits a hexagonal ordering of spots, with a typical wavelength of the order of $0.5 \mathrm{~mm}$ and oscillating in amplitude with a period of the order of $5 \mathrm{~min}$. The pixel size and refreshing time of the noise were chosen, respectively, to be $0.1 \mathrm{~mm}$ and $15 \mathrm{~s}$, large enough to produce a sizeable effect but small compared with the given space and time scales of the respective Turing and Hopf modes.

In a typical experiment, the system entrains, after a transient, to a rhythm of synchronized oscillating spots [13]. After a few periods of the sustained oscillation of the spots, the noise is switched on. Following noisy forcing, oscillations are drastically arrested within one to two periods. Only some minor incoherent and irregular twinkling of the spots remains as a residual dynamics of the otherwise perfectly steady pattern. Different representations of this phenomenon are shown in fig. 2. Noise intensities must be carefully selected, since spots are either unaffected or incoherently created and destroyed under, respectively, too weak or strong random forcing.

Two-dimensional simulations were conducted using the LE model [15] modified to account for light sensitivity [9]

$$
\begin{gathered}
\frac{\partial u}{\partial t}=a-c u-\frac{4 u v}{1+u^{2}}-\phi(x, t)+\nabla^{2} u, \\
\frac{\partial v}{\partial t}=b\left(c u-\frac{u v}{1+u^{2}}+\phi(x, t)+d \nabla^{2} v\right) .
\end{gathered}
$$

In this dimensionless form, $u$ and $v$ are proportional to the activator $\left(\mathrm{I}^{-}\right)$and inhibitor $\left(\mathrm{ClO}_{2}^{-}\right)$concentrations; $a$, $b$, and $c$ are parameters related to other initial concentrations and rate constants, and $d$ is proportional to the ratio of diffusivities. $\phi(x, t)$ stands for the illumination intensity. To mimic the experiments above, we take $\phi(x, t)=$ $\phi_{0}+\xi(x, t)$, with $\xi(x, t)$ an additive, Gaussian, zero-mean $\left(\left\langle\xi_{i}(t)\right\rangle=0\right)$ random quantity, independently prescribed in each lattice site and following an Ornstein-Uhlenbeck statistics $\left\langle\xi_{i}(t) \xi_{j}\left(t^{\prime}\right)\right\rangle=(\epsilon / \tau) \exp \left(-\left|t-t^{\prime}\right| / \tau\right) \delta_{i j}$. This set of stochastic reaction-diffusion equations was solved using a standard 5-points discretization, with no-flux boundary conditions, and the Heun method for temporal integration $[20]$.

In the absence of superimposed noise we characterize the LE model in terms of a phase diagram constructed from a linear stability analysis around the unstable rest state $\left(u_{0}=(a-5 \phi) / 5 c\right.$ and $\left.v_{0}=\left(25 c^{2}+(a-5 \phi)^{2}\right) a / 25 c(a-5 \phi)\right)$. The basic results are summarized in fig. 3. Beyond both the Turing and Hopf instabilities, but still close to the latter bifurcation curve, (fig. 3A), the oscillatory mode is weak and Turing patterns dominate, except near the Turing/Hopf cobifurcation conditions where oscillating spots are observed (point B). Simulations slightly beyond that point show, conversely, a large number of mixed modes and complex dynamics, for example bistability, formation of moving domains between two modes or transient solutions [21].

As a first numerical experiment to compare with the CDIMA results under noise forcing, we consider the system initially free of external noise and with a random distribution of the $u$ and $v$ variables around their values at the center of the limit cycle $\left(u_{0}, v_{0}\right)$. A proper choice of model parameters (see caption of fig. 4) leads, in absence of external noise, to a pattern of oscillating spots [13]. 

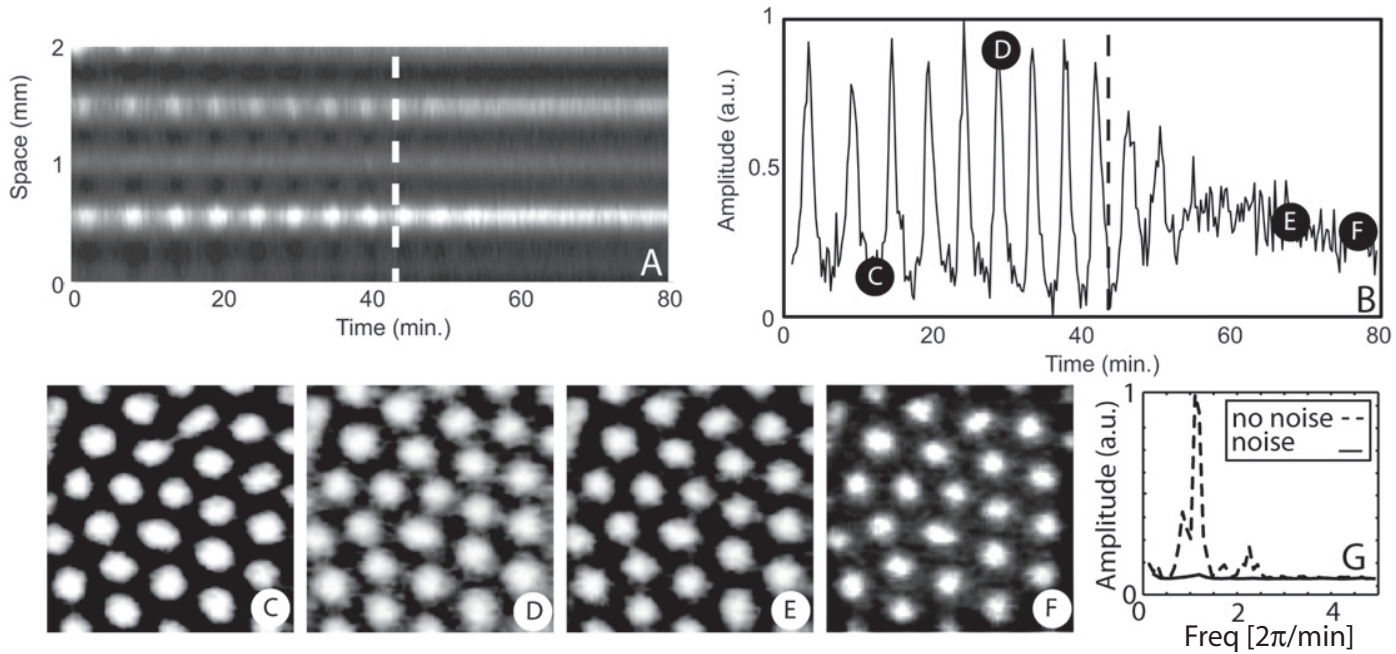

Fig. 2: Effect of noise over an experimental oscillatory Turing pattern. (A) Space-time plot constructed from a one-dimensional cut of the original two-dimensional lattice of oscillating spots. The dashed line marks the time when noise is introduced. (B) Gray intensity as a measure of inhibitor concentration in arbitrary units (a.u.), averaged over an area of seven spots. Once noise is applied, synchronized oscillations are largely suppressed. The horizontal axis (time) is the same in both plots. Letters inserted in (B) refer to the corresponding snapshots $\left(2 \times 2 \mathrm{~mm}^{2}\right)$ of the experiment: (C) lower phase of oscillation, (D) higher phase of oscillation, (E-F) twinkling pattern under noisy forcing. (G) The Fourier transform of panel B for both cases with and without noise is compared.

A

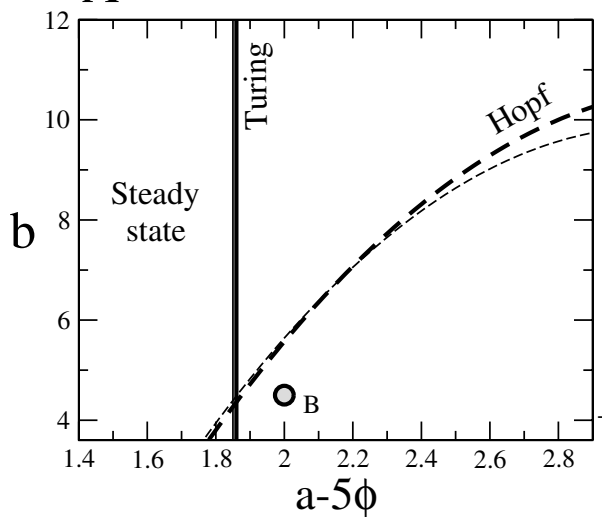

B

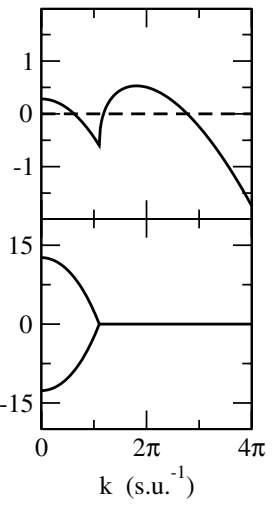

Fig. 3: A) Phase diagram of the LE model for fixed parameters $c=0.3, d=1.07$. Thick lines correspond to $\phi=3.2$ and thin lines to $a=18$. Vertical solid lines correspond to the Turing bifurcation and curved dashed lines to the Hopf bifurcation. Panel B shows the dispersion relation for real (above) and imaginary (below) components of the eigenvalues obtained with a linear stability analysis corresponding to conditions marked $\mathrm{B}$ in panel A. Point B was chosen to correspond to numerical simulations in figs. 4,5 and 6.

Again, when external noise is introduced into the model, the oscillations are largely attenuated (see fig. 4), as it is observed experimentally, although some irregular twinkling of the spots is here slightly more apparent than in experiments.

Second panels in fig. 2 and fig. 4 show the evolution of a local quantity, the amplitude of the activator variable integrated over an area containing seven spots. The
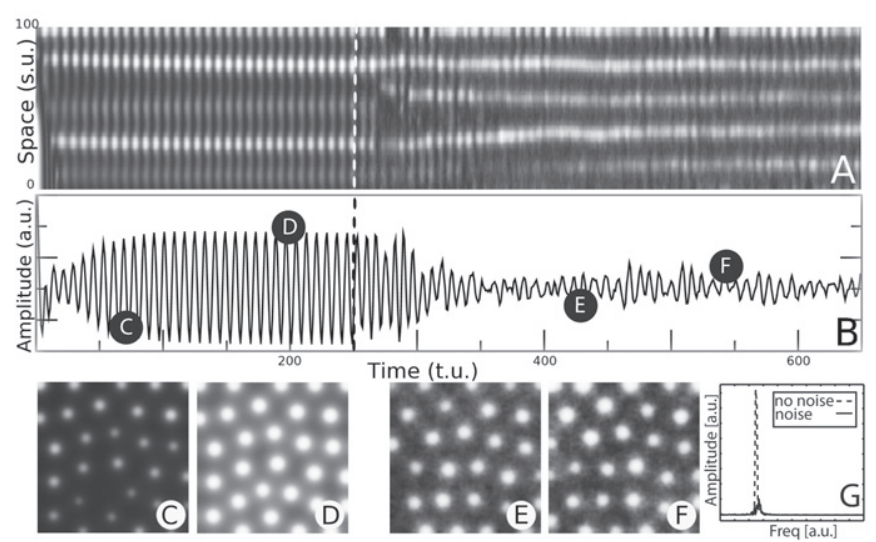

Fig. 4: Effect of noise over a numerically simulated oscillatory Turing pattern. (A) Space-time plot in the LE model with parameter values $a=18.0, c=0.3, b=4.5, \phi=3.2$ and $d=1.07$. Temporal and spatial discretization units are, respectively, $\Delta t=0.0025$ time units (t.u.) and $\Delta x=0.5$ space units (s.u.). The dashed line marks the time when the noise is introduced with intensity $\epsilon=0.03$ and correlation time $\tau=0.01$ t.u. (B) Gray intensity (as a measure of inhibitor concentration) averaged over a circle of radius $10 \mathrm{s.u}$. Once noise is applied, synchronized oscillations are largely arrested. Panels (C) and (D) correspond, respectively, to lower and higher phase of oscillation. (E-F) Snapshots after noise forcing, with a phase separation of half of the deterministic period ( $T_{0}=9.7$ t.u.). The displayed region corresponds to $50 \times 50 \mathrm{s.u}^{2}$. (G) The Fourier transform of panel $\mathrm{B}$ for both cases with and without noise is compared.

attenuation of the amplitude of the oscillation by the noise is clearly observed in both cases. To confirm that the external noise indeed attenuates the oscillation, rather than 


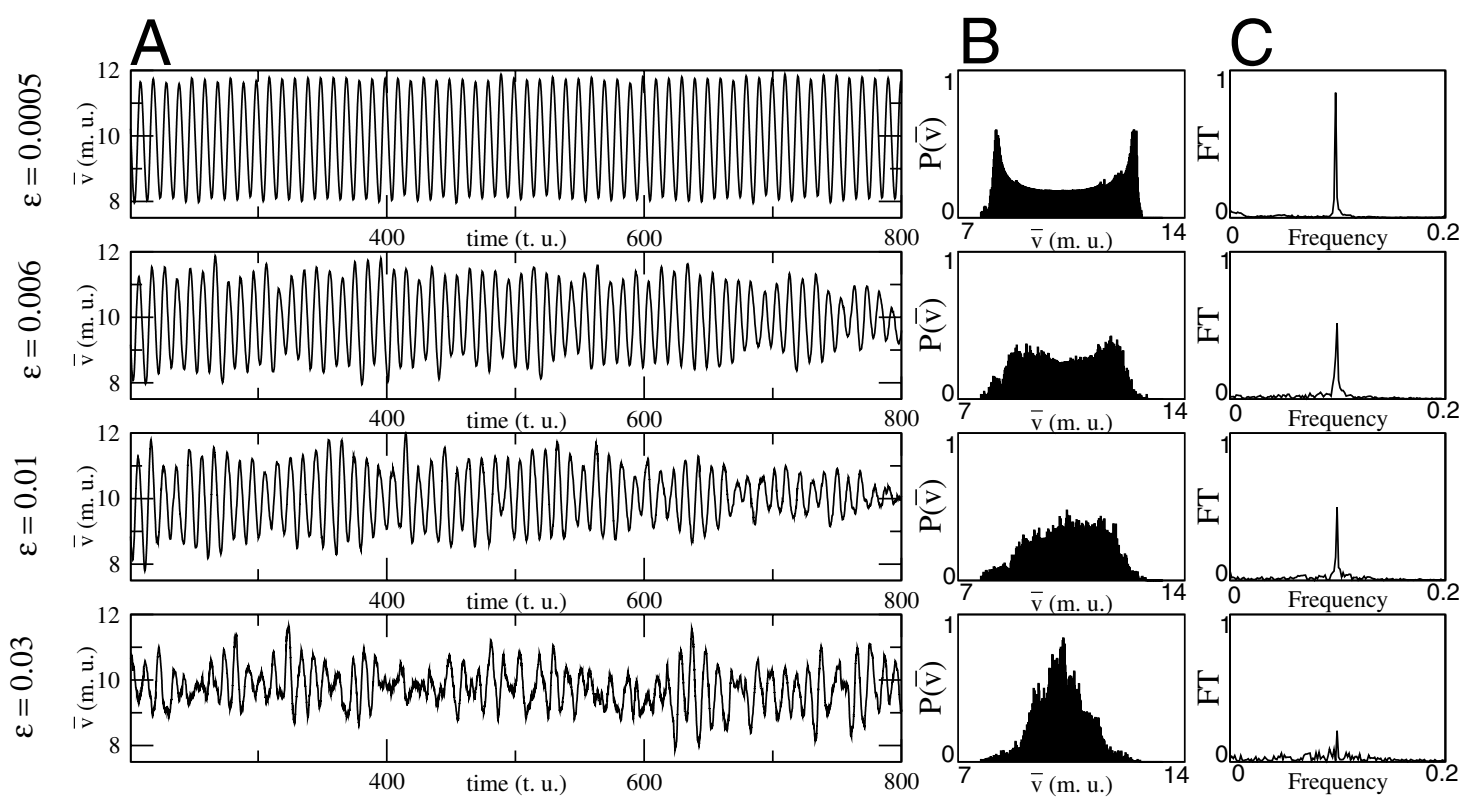

Fig. 5: Effects of superimposed noise on the LE model for the dynamics of a single spot under conditions marked B in fig. 3 (oscillating spots). Registers correspond to four different noise intensities. Parameter values as in fig. 4. Plots in column A shows the temporal trace of the averaged amplitude for a single spot. Plots in column B shows the probability distribution of such averaged amplitude. Fourier transforms (FT) of the signals in A are calculated and plotted in column C. The units of the concentrations are adimensional and are noted by model units (m.u.). Units of panels in columns B and C are arbitrary.

causing a simple spatial desynchronization of the spots, we proceed to analyze the amplitude change of the oscillation over a single spot. This study is experimentally unfeasible due to limited accuracy but can be simply performed on numerical simulations. During the numerical integration of eqs. (1) we monitor a particular spot and compute an averaged amplitude of the inhibitor over the whole area of that spot

$$
\bar{v}=\frac{1}{A} \int_{x_{o}-x_{m}}^{x_{o}+x_{m}} \mathrm{~d} x \int_{y_{o}-y_{m}}^{y_{o}+y_{m}} \mathrm{~d} y v(x, y) .
$$

In the latter expression $\left(x_{o}, y_{o}\right)$ corresponds to the local maximum of the inhibitor field, calculated at each temporal step, $x_{m}$ and $y_{m}$ are chosen to encompass the whole spot, and $A$ denotes the total integrated area. The evolution of this quantity is plotted in fig. 5A. When the noise is very weak (first row on fig. 5) the oscillation of a single spot is perfectly periodic and the corresponding probability of finding a particular value of the amplitude $P(\bar{v})$ displays the characteristic shape of a sinusoidal signal, with two peaks corresponding to the extrema of the oscillation. The Fourier representation displays the characteristic peak at the oscillating frequency. The introduction of noise makes the spot oscillations to loose temporal coherence (second and third rows on fig. 5) and the probability distribution correspondingly flattens. For a moderate intensity of the noise (last row on fig. 5) the spots behave much more irregularly and the probability distribution tends to adopt a Gaussian shape centered at the midpoint between the original maxima. The Fourier transform still peaks at the oscillation frequency but with a substantially decreased strength. The oscillation of the spots has been practically arrested and it is thus being replaced by a random dynamics.

A more systematic analysis is performed by calculating the standard deviation $(\sigma)$ of the probability distribution $P(\bar{v})$, plotted in fig. 5B, and the intensity of the Fourier peak $\left(v_{\omega_{o}}\right)$, plotted in fig. $5 \mathrm{C}$. To gain a confident statistics we follow five different spots in each numerical simulation and the procedure is four times repeated with different random initial conditions and different realizations of the noise. In fig. 6 we plot the averaged values $\bar{\sigma}$ and $\overline{v_{\omega_{o}}}$ for a wide range of noise intensities. For very low noise amplitudes the standard deviation is quite large as corresponds to the harmonic periodic signal. As the noise intensity increases, the standard deviation gets reduced due to the change on the shape of the distribution $P(\bar{v})$ (see fig. 5B). After this reduction, it passes through a minimum because the Gaussian-like distribution $P(\bar{v})$ gets wider with higher noise intensities fig. $6 \mathrm{~A}$. It is thus clear that there is a particular noise intensity for which the reduction of the local oscillation of the spots is maximal, as expected. Eventually, for intense noise the spots are randomly created and destroyed. This regime further enhances the standard deviation that is now the signature of a completely noisy signal. Such dynamics is observed for $\epsilon>0.08$ and it is not shown in fig. 6 . In terms of the maximum response in Fourier spectrum, attenuation of 

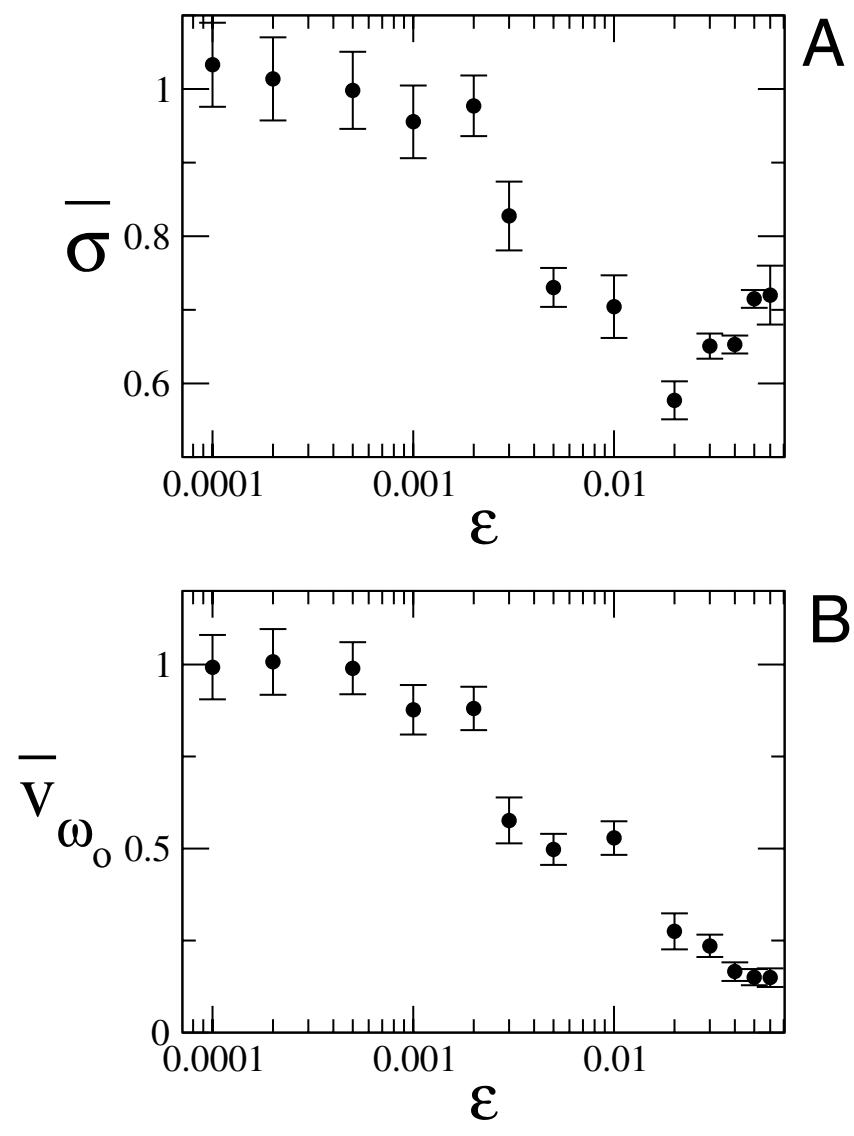

Fig. 6: Study of the effects of superimposed noise on the mixed mode solution of the LE model in the situation described in fig. 5. The mean standard deviation of the probability distribution (column B of fig. 5) and the strength of the peak of the Fourier transform (column C of fig. 5) are plotted for different noise intensities. The latter quantity is renormalized to the corresponding value of the system in the absence of superimposed noise. For each intensity an average over twenty spots is employed (see text). Error bars in both magnitudes account for statistical errors. Parameter values as in fig. 4.

oscillations is also clearly seen, reproducing at the highest noise intensities the quasi-flat spectrum of the forcing random signal.

Numerical studies concerning the effects of the correlation time $(\tau)$ does not show substantial differences with respect to the results obtained here in the limit of small $\tau$. In particular, frozen noise is also able to arrest oscillations. Contrarily such an effect is not observed when increasing the correlation length. Actually for uniform noise (only time dependent) the Turing pattern is destroyed in this case and the system recovers a regime of global Hopf-like oscillations.

The phenomena here reported point out to a noiseenhanced relative stability of the Turing vs. the Hopf mode. One might thus be tempted to propose an interpretation based on a noise-mediated renormalization of the LE parameters, eventually leading to a shift of the stability boundaries in the deterministic phase diagram (fig. 3). This idea, much invoked in relation to multiplicative-noise effects [7], cannot be completely ruled out in the present additive case, since fluctuations enter into the LE model simultaneously for the activator/ inhibitor couple (see eq. (1)) [17]. In any case, this approach would require a challenging theoretical elaboration, including noise correlation effects, that goes beyond the scope of the present account. The possibility, instead, of using reduced, amplitude-like equations, is suggestive but again non-standard when noise with spatiotemporal structure acts on a (spatial and temporal) cobifurcation, as it is the case here. We thus propose at this time a more intuitive interpretation based on a different susceptibility of the coexisting Turing and Hopf modes. A global oscillation, either homogeneous or mixed with a patterned mode as it is considered here, supposes a high degree of spatial synchronization, entirely mediated by diffusion. Facing distributed noise of intermediate intensity, such condition may be rather vulnerable to rapidly changing fluctuations of small correlation length. The underlying Turing solution may benefit from its intrinsic noise-averaging nature at these scales and thus appears more robust in front of fast evolving fluctuations of small intensity and whose spatial structure does not exceed the pattern wavelength.

In summary, we have studied the effect of random forcing on the CDIMA reaction near a Hopf/Turing bifurcation. Prevalence of the latter mode is evidenced for noise of small correlation parameters and moderate intensity. To eventually probe the generality of this phenomenon in other scenarios of mixed bifurcations, different from the chemical context here, i.e. semiconductor [22] or optical devices [23], should be a challenge for future experimental and theoretical research.

Experiments were carried out in Santiago de Compostela, Spain. Financial support was provided by Ministerio de Educación y Ciencia (Spain) under project FIS2006-03525 (FS), by DURSI though project 2005-SRG/00653 (FS) and by E.U. Network "Unifying principles in nonequilibrium pattern formation" (SA). DGM acknowledges the postdoctoral fellowship EX-20060420. Special thanks to A. P. Muñuzuri for useful experimental advices and suggestions.

\section{REFERENCES}

[1] Sagués F., Sancho J. M. and García-Ojalvo J., Rev. Mod. Phys., 79 (2007) 829.

[2] Pikovsky A. S. and Kurths J., Phys. Rev. Lett., 78 (1997) 775.

[3] Goldman D. I., Swift J. B. and Swinney H. L., Phys. Rev. Lett., 92 (2004) 174302.

[4] Sagués F. and Epstein I. R., Dalton Trans., issue No. 7 (2003) 1201. 
[5] Sendiña-Nadal I. et al., Phys. Rev. Lett., 84 (2000) 2734.

[6] KÁdÁR S., Wang J. C. and Showalter K., Nature, 391 (1998) 770.

[7] Alonso S., Sendiña-Nadal I., PÉrez-Muñuzuri V., SAncho J. M. and SAguÉs F., Phys. Rev. Lett., 87 (2001) 078302.

[8] Castets V., Dulos E., Boissonade J. and De Kepper P., Phys. Rev. Lett., 64 (1990) 2953.

[9] Muñuzuri A. P., Dolnik M., Zhabotinsky A. M. and Epstein I. R., J. Am. Chem. Soc., 121 (1999) 8065.

[10] Rüdiger S., Míguez D. G., Muñuzuri A. P., Sagués F. and Casademunt J., Phys. Rev. Lett., 90 (2003) 128301.

[11] Míguez D. G. et al., Phys. Rev. Lett., 93 (2004) 048303.

[12] Míguez D. G., Pérez-Villar V. and Muñuzuri A. P., Phys. Rev. E, 71 (2005) 066217.

[13] Míguez D. G., Alonso S., Muñuzuri A. P. and Sagués F., Phys. Rev. Lett., 97 (2006) 178301.
[14] Sanz-Anchelergues A., Zhabotinsky A. M., Epstein I. R. and Muñuzuri A. P., Phys. Rev. E, 63 (2001) 056124 .

[15] Lengyel I. and Epstein I. R., Science, 251 (1991) 650.

[16] Carrillo O., Santos M. A., García-Ojalvo J. and SANCHO J. M., Europhys. Lett., 65 (2004) 452.

[17] Riaz S. S., Dutta S., Kar S. and Ray D. S., Eur. Phys. J. B, 47 (2005) 255.

[18] Buceta J. and Lindenberg K., Phys. Rev. E, 66 (2002) 046202 .

[19] Sailer X., Hennig D., Beato V., Engel H. and Schimansky-Geier L., Phys. Rev. E, 73 (2006) 056209.

[20] García-Ojalvo J. and Sancho J. M., Noise in Spatially Extended Systems (Springer, New York) 1999.

[21] De Wit A., Lima D., Dewel G. and Borckmans P., Phys. Rev. E, 54 (1996) 261.

[22] Just W., Bose M., Bose S., Engel H. and Schöll E., Phys. Rev. E, 64 (2001) 026219.

[23] Paulau P. V., Babushikin I. V. and Loiko N. A., Phys. Rev. E, 70 (2004) 046222. 